\title{
Touch-Based System for Beat-to-Beat Impedance Cardiogram Acquisition and Hemodynamic Parameters Estimation
}

\author{
Dionisije Sopic $^{1}$, Srinivasan Murali $^{2}$, Francisco Rincón ${ }^{1,2}$, David Atienza ${ }^{1}$ \\ ${ }^{1}$ Embedded Systems Lab. (ESL), EPFL, Switzerland, ${ }^{2}$ SmartCardia, Switzerland \\ \{dionisije.sopic, francisco.rincon, david.atienza\}@epfl.ch, srinivasan.murali@ smartcardia.com
}

\begin{abstract}
Among all cardiovascular diseases, congestive heart failure (CHF) has a very high rate of hospitalization and mortality. In order to prevent hospitalization, there is a strong need to identify patients at risk of a CHF event by estimating a set of relevant hemodynamic parameters that will allow physicians to detect its early onset. Today, one of the most popular non-invasive methods to obtain these parameters is through the acquisition of electrocardiogram (ECG) and impedance cardiogram (ICG) by using large hospital systems with electrodes placed on the chest and thorax region. In order to be useful in an ambulatory setting, it is important to obtain an ultra-low power wearable system for acquiring the ICG and ECG, and to detect CHF. In this paper, we present a touch-based ultra-low power device for real-time ICG and ECG signal acquisition, and hemodynamic parameters estimation. We also propose methods for noise cancellation and for calculating the hemodynamic parameters. In addition, a comparative evaluation of susceptibility to different measuring positions is presented. Our proposed design is highly correlated with traditional systems $(>80 \%)$, but able to work with very low power budgets, thus allowing long duration of operation of over four days on a single battery charge.
\end{abstract}

\section{INTRODUCTION}

Congestive heart failure (CHF) is one of the major public health issues, and is characterized by frequent hospital admissions and high mortality rates. It has been estimated that annually in the United States alone, nearly 5 million patients are affected by CHF [1]. CHF is a condition in which the heart fails to pump enough blood to meet the body's needs impeding the proper cells' nourishment. It is usually preceded by an increase of fluid in the thoracic cavity. In addition, it is accompanied by swelling of limbs, shortness of breath, quick weight gain as well as irregular heartbeat. Many studies have been conducted in an attempt to detect early onset of CHF. For instance, in [2], the authors indicate that, on average, weight gain is an important risk factor preceding the hospitalisation within a relatively short period, but their results do not indicate that all hospitalizations are preceded by weight gain. Furthermore, in order to prevent hospitalization, there is a need to identify patients at risk of a major heart failure event by estimating some more relevant and more reliable parameters. Hemodynamic parameters can be used as relevant parameters for detecting an early onset of CHF, a particularly important time when CHF can still be prevented by change of medication [3]. These parameters are used to determine how efficiently the heart pumps blood through the

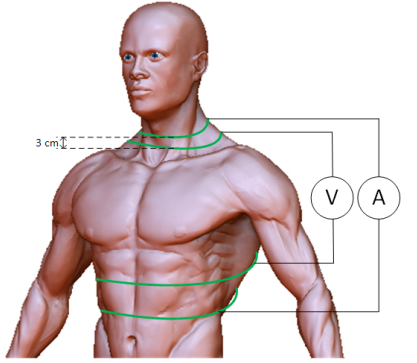

Fig. 1: Traditional electrode configuration setup. [5] cardiovascular system. Determining them is of the utmost importance as the proper blood circulation is a necessary condition for adequate supply of oxygen to all tissues. Gold standard techniques used for these purposes are invasive, and involve many risks [4]. For these reasons, in the last two decades several non-invasive methods have been proposed to monitor cardiac hemodynamics.

We focus on Impedance cardiogram (ICG), which is a key technique for non-invasive methods in assessing these hemodynamic parameters as well as the fluid level status. ICG measurement process is usually conducted by four electrodes, (Fig. 1). In this process, an alternating current (AC) of low amplitude is injected across the human body through the outer electrodes, and the voltage is measured through the inner ones. ICG has usually been recorded by large systems, by means of cables connected to electrodes, which are placed on the chest, permitting the current injection. Thus, today's ICG acquisition methods are not conducive for point-of-care use by patients, wherein the hemodynamic parameters can be measured quickly and conveniently.

In this paper, we present a touch-based ultra-low power device, easy to use as shown in Fig. 2, which allows realtime ICG and ECG signals acquisition by touch. The main contributions of this paper are the following:

1) A point-of-care device for beat-to-beat ECG and ICG signal acquisition from fingers, which includes algorithms and methods for embedded real-time ECG and ICG filtering.

2) Showing the correlation between signals obtained from our touch-based low-power device and those obtained through the traditional method for ICG signal acquisition which involves the placement of the electrodes on the human's body. 


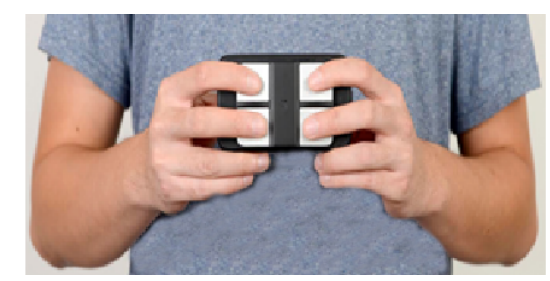

Fig. 2: Touch-based ultra-low power device

3) Quantifying the error which occurs due to device displacement during measurements.

4) Methods to obtain relevant hemodynamic parameters.

This paper is organized as follows. Previous work on ICG is described in Section 2. The design methodology used in this study is presented in Section 3. Finally, experiments and results are shown in Section 4, whilst conclusions are discussed in Section 5.

\section{RELATED WORK}

Several studies have been recently conducted in an attempt to investigate the usefulness of ICG as a reliable and noninvasive technique for body composition assessment as well as for disease prediction. In [6], the authors showed that ICG could be used as an accurate and reproducible alternative to invasive techniques for measuring hemodynamic parameters in patients with ischemic cardiomyopathy, either at rest or during upright exercise. Furthermore, in [7] it has been shown that ICG can identify patients at highest and lowest short-term risk for a heart failure event. One of the methods for peripheral and pulmonary edema detection was described in [8]. A study which uses ICG in order to obtain valid and reliable estimates of cardiac function in persons with hypertension is described [9]. Thoracic fluid content measured by ICG has been used as a parameter for predicting beneficial effects of cardiac rehabilitation in patients suffering from CHF in [10]. ICG has also been studied in the evaluation of the hemodynamic responses during psychological stress in [11].

Some of the commercial devices for ICG signal acquisition are Philips ICG monitor [12] and LIFEGARD II MultiParameter Patient Monitor [13]. A measuring board based on low-power ECG and ICG is proposed in [14]. In order to acquire signals, these devices use electrodes placed on the neck and on the thorax, whereas our proposed system operates simply by fingers' touch.

ICG signal is usually contaminated by two main artifacts: respiratory and motion artifacts. ICG signal spans the frequency range $(0.8-20) \mathrm{Hz}$, while respiratory and motion artifacts have frequency components in $(0.04-2) \mathrm{Hz}$ and $(0.1-10) \mathrm{Hz}$, respectively [15]. Different techniques for ICG artifacts suppression have been reported in the literature [16], [17].

\section{Hardware Design Methodology}

The system used in this experiment is shown in Fig. 2. This device features a sensor which enables obtaining both ECG and ICG signals at the same time in a continuous

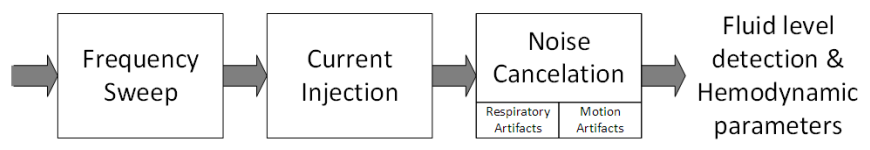

Fig. 3: Proposed algorithm flowchart for ICG and ECG signal acquisition

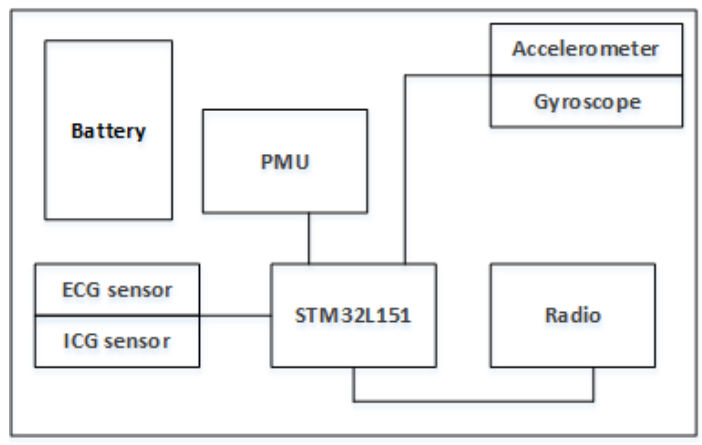

Fig. 4: Block diagram of the reference node architecture manner, just by touch. The frequency of the injected current for ICG measurements, as well as the sampling frequency of the physiological signals are adjustable.

\section{A. Hardware architecture}

- The ECG and ICG sensor describes the hardware component that acquires and samples the raw signal. Then, the samples are quantized by an A/D converter using a number of bits that depends on its resolution. Sampling frequency goes from $125 \mathrm{~Hz}$ up to $16 \mathrm{Khz}$ with up to 16bits resolution. We use a standard ECG frontend (ADS1291) [18] and a proprietary sensor for ICG acquisition.

- The STM32L151 [19] is an ultra-low power 32-bit microcontroller which can operate at a maximum frequency of $32 \mathrm{MHz}$, has $48 \mathrm{~KB}$ RAM, $384 \mathrm{~KB}$ Flash and analog peripherals including a 12-bit ADC.

- The accelerometer and gyroscope sense motion, which are used for to distinguish different positions.

- The radio describes the hardware used to transmit the data though the wireless channel. We use Bluetooth low energy (nRF8001) [20].

- The Power Management Unit (PMU) dynamically tunes the system to achieve the best trade-off between energy consumption and performance, taking into account the available energy in the battery and requirements (accuracy, latency, etc.) of the target application.

The current consumed by each of the previous component is presented in Table I.

\begin{tabular}{c|c} 
Component & Average current (mA) \\
\hline ECG chip & 0.400 \\
\hline ICG chip & 0.900 \\
\hline STM32L151 (active) & 10.500 \\
\hline STM32L151 (standby) & 0.020 \\
\hline Radio (TX) & 11.000 \\
\hline Radio (standby) & 0.002 \\
\hline Gyroscope + Accelerometer & 3.800 \\
\hline
\end{tabular}

TABLE I: Current consumption for each component 


\section{SOFTWARE DESIGN METHODOLOGY}

The proposed algorithm flowchart for ICG and ECG signal acquisition is depicted in Fig. 3. As this figure shows, we first set the frequency of the current that we inject in the human body. After passing it across the human body and obtaining the developed voltage, methods for noise cancellation are applied and hemodynamic parameters along with the fluid level are detected.

\section{A. Noise cancellation}

In this work, we have developed methods that can run in real-time on the embedded platform. We have used the following methods for noise reduction:

1) ECG filtering:

- Baseline wander removal through morphological filtering [21]: This method applies several erosion and dilation operations to the original ECG signal to estimate the baseline wander. It first applies an erosion followed by a dilation, which removes peaks in the signal. Then, the resultant waveforms with pits are removed by a dilation followed by an erosion. The final result is an estimate of the baseline drift. The correction of the baseline is then done by subtracting this estimate from the original signal.

- Zero-phase band-pass filter for high-frequency noise and artifact removal: A 32nd-order FIR bandpass filter with cut-off frequencies $f_{1}=0.05 \mathrm{~Hz}$ and $f_{2}=40 \mathrm{~Hz}$ is used for these purposes.

2) ICG filtering: After looking at the frequency spectrum of the signal, we noticed that amplitudes of the components at frequencies $f>20 \mathrm{~Hz}$ were not significant in comparison with those obtained at lower frequencies. Therefore, to minimize any kind of high-frequency noise interference, we use a zerophase low-pass Butterworth filter with cut-off frequency $f=$ $20 \mathrm{~Hz}$.

\section{B. ICG characteristic points}

After applying an alternating electric current across the human body (Fig. 1), we first get the thoracic bioimpedance, $Z_{0}$. Analysis of bioimpedance signal assumes that the resistance to electric current is inversely proportional to the distribution of body water and electrolytes. Lean tissues are high conductors of electric current due to their large amount of water and electrolytes, and therefore, they show low resistance to the passage of a current. On the other side, fat and bone have low conductivity and, with a smaller quantity of fluids and electrolytes, they show high electrical resistance [22]. ICG signal is then calculated as:

$$
I C G=-\frac{d Z}{d t}
$$

A typical ICG waveform along with an ECG waveform is depicted in Fig. 5. Points $\mathrm{B}, \mathrm{C}$ and $\mathrm{X}$ are the three main characteristic points of the ICG signal. Point B represents the opening of the aortic valve, while point $\mathrm{X}$ denotes its closure [23]. Changes in the ICG are generated by fluctuations in blood volume and flow velocity in the ascending aorta during each heart cycle. The time interval between point $\mathrm{B}$ and point

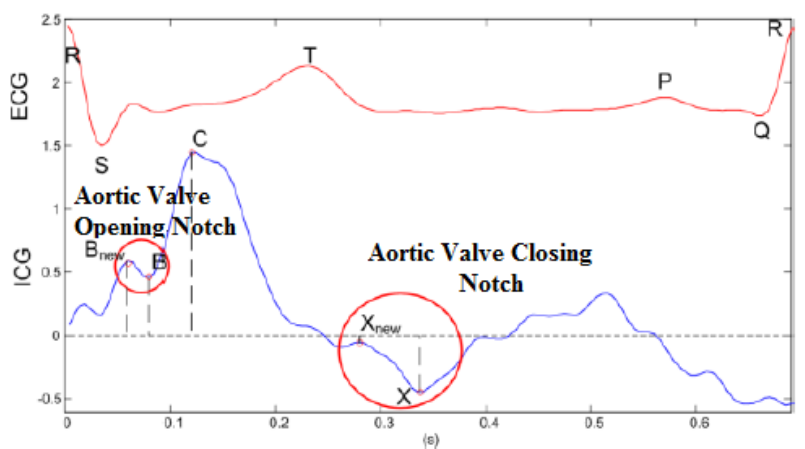

Fig. 5: ICG and ECG waveform (cf. [28])

$\mathrm{X}$ is the Left Ventriclar Ejection Time (LVET) while the time interval between R-wave at the ECG and B point at the ICG is the Pre-Ejection Period (PEP) [24]. LVET gives information about how long it takes for the heart to pump blood out of the left ventricle. PEP represents the electrical-mechanical delay that occurs from the onset of depolarization to the beginning of the ventricular contraction, that is when the aortic valve opens. These parameters are called systolic time intervals and they are used to estimate cardiac output (CO) and stroke volume (SV) [25], [26]. For calculating LVET and PEP, the frequency of the injected current was set to $f=50 \mathrm{Khz}$, considering the work done in [27].

\section{Algorithm used for ICG characteristic point detection}

The algorithm operates on a beat-to-beat basis and is based on [28]. As our device is acquiring ECG and ICG simultaneously, R peaks are detected by using Pan-Tompkins algorithm [29]. After that, ICG signal included between two consecutive ECG R-peaks was fed into the algorithm.

- $C$ point - corresponds to the maximum value of the ICG signal.

- B point - First the initial point B0 is estimated. B0 is obtained based on the intersection of the line fit of the ICG points between $40 \%$ and $80 \%$ of the amplitude of point $\mathrm{C}$ with the horizontal axis. If the $(+,-,+,-)$ sign pattern of the second order derivative of ICG to the left of the C-Point is detected, the B-point is defined by the first minimum of the 3rd derivative to the left of B0. If no sign pattern in the second derivative of ICG is detected, the B-point is assumed to be the first zero-crossing of the first derivative of the ICG to the left of B0. Point $B_{\text {new }}$ in Fig. 5 represents the opening of the aortic valve used in our algorithm, whereas the point $B$ represents its traditional definition mostly used in the literature.

- $X$ point - As an initial estimate of $X$ point, the lowest ICG negative minimum to the right of $\mathrm{C}$ point is searched (X0). Point $\mathrm{X}$ is detected as the local minimum of the 3rd derivative of the ICG to the left of (X0). It should be mentioned that in [28], authors proposed a 
slightly different approach for finding the initial estimate for X point. Namely, in their study the closing of the aortic valve is usually observed near the end of the T-wave. Therefore, they are taking the lowest ICG negative minimum in the interval $R T \leq t \leq 1.75 R T$ as (X0), where RT represents the duration of the R-T ECG segment. In their algorithm, the closure of the aortic valve is represented as point $X_{n e w}$, Fig. 5. The main reason we have adjusted the algorithm is the well-known fact that the end of a $\mathrm{T}$ wave is not a reliable marker and it is not very clear where this wave ends. Point $X$ in Fig. 5 represents the closure of the aortic valve most often reported in the literature.

\section{EXPERIMENTS AND RESULTS}

Five male subjects took part in this experiment. First, we have recorded the ICG signal from our subjects using the traditional method for signal acquisition by placing the electrodes on the chest and thorax, Fig. 1. Depending on the frequency of the applied current, the path of the current may change [30]. At low frequency $(f<50 \mathrm{Khz})$, the current does not penetrate the cell membrane, and the current passes through the extracellular fluid. At a very high frequency $(f \geq 50 \mathrm{Khz})$, the current passes just through both, extracellular and intracellular fluid. Due to this, signals were recorded for four different frequencies: $f \in\{2,10,50,100\} \mathrm{Khz}$. Sampling frequency of ECG and ICG signals was set to $250 \mathrm{~Hz}, f_{s}=250 \mathrm{~Hz}$.

In our experiment, we investigate three different positions of subjects' arms during our measurements. Our goal is to identify the position that has the best correlation with the traditional measuring setup shown in Fig. 1. Moreover, we want to quantify the worst measurement error that might occur when the user's arm is displaced from the intended position during the measurement process. The subjects were required to be standing in each of the positions. In Position 1, they were asked to hold the device up to their chest. Then, they were asked to stretch out their arms in front of them parallel to the floor (Position 2). Finally, subjects were asked to slowly put their arms down by their sides (Position 3). We chose these positions to find the worst-case error that can occur if users move the device during the measurement. Signals were recorded for 30 seconds in each of the positions for each of the four frequencies of the injected current.

Then, we analyzed the average thoracic bioimpedance $\left(Z_{o}\right)$. The bioimpedance signal obtained through the traditional electrode setup (Fig. 1) is shown in Fig. 6, whereas figures 7a, $7 \mathrm{~b}, 7 \mathrm{c}$ represent the mean value of the bioimpedance signal obtained from our device. From these figures, we can notice the same behavior of the signals obtained from our device and those obtained through the traditional electrode setup (Fig. 1). Namely, the bioimpedance signal increases until $f=10 K h z$, and then it starts decreasing. The correlation between the signals obtained through the traditional setup (Fig. 1) and those obtained from our device is tabulated in Tables II, III, IV. According to our results, the lowest overall correlation is obtained in Position 3.

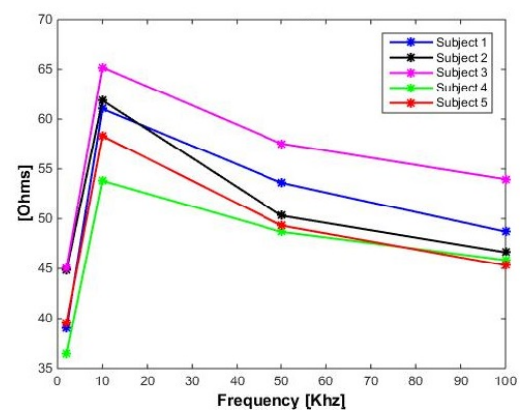

Fig. 6: Thoracic Bioimpedance

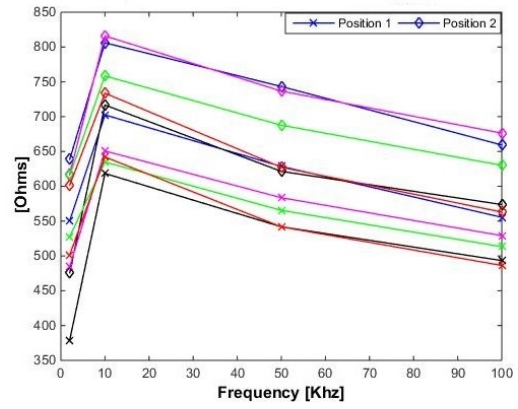

(a) Positions $1 \& 2$

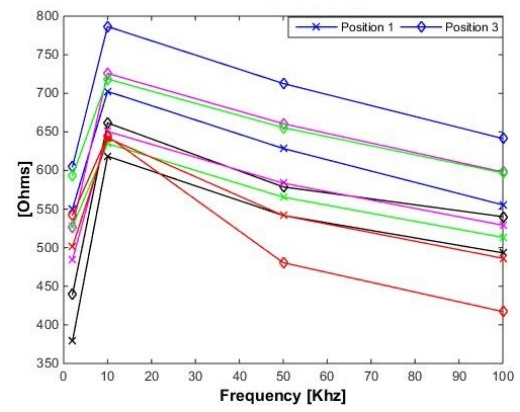

(b) Positions $1 \& 3$

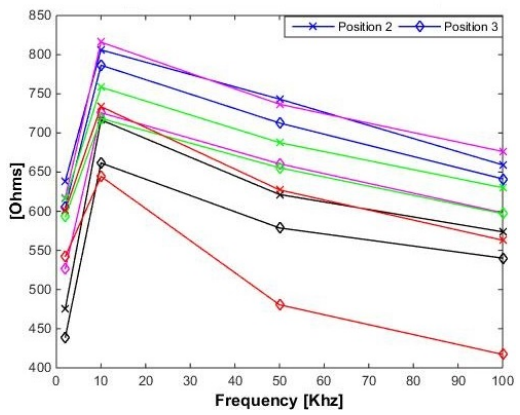

(c) Positions $2 \& 3$

Fig. 7: Bioimpedance signals obtained from the device

We introduce the relative error as a criteria for defining the worst-case error due to movements of the device during the experiment, equations $1,2,3$.

$$
e_{21}=\frac{Z_{\text {position }_{2}}-Z_{\text {position }_{1}}}{Z_{\text {position }_{2}}}
$$




\begin{tabular}{|c|c|}
\hline Subjects & Correlation Coefficient \\
\hline Subject 1 & 0.9081 \\
\hline Subject 2 & 0.9471 \\
\hline Subject 3 & 0.9827 \\
\hline Subject 4 & 0.8451 \\
\hline Subject 5 & 0.9251 \\
\hline
\end{tabular}

TABLE II: Correlation Position 1 VS Thoracic bioimpedance

\begin{tabular}{|c|c|}
\hline Subjects & Correlation Coefficient \\
\hline Subject 1 & 0.9747 \\
\hline Subject 2 & 0.9497 \\
\hline Subject 3 & 0.9938 \\
\hline Subject 4 & 0.9033 \\
\hline Subject 5 & 0.8461 \\
\hline
\end{tabular}

TABLE III: Correlation Position 2 VS Thoracic bioimpedance

\begin{tabular}{|c|c|}
\hline Subjects & Correlation Coefficient \\
\hline Subject 1 & 0.9737 \\
\hline Subject 2 & 0.9377 \\
\hline Subject 3 & 0.9908 \\
\hline Subject 4 & 0.8531 \\
\hline Subject 5 & 0.6919 \\
\hline
\end{tabular}

TABLE IV: Correlation Position 3 VS Thoracic bioimpedance

$$
\begin{aligned}
& e_{23}=\frac{Z_{\text {position }_{2}}-Z_{\text {position }_{3}}}{Z_{\text {position }_{2}}} \\
& e_{31}=\frac{Z_{\text {position }_{3}}-Z_{\text {position }_{1}}}{Z_{\text {position }_{3}}}
\end{aligned}
$$

$Z_{\text {position }_{x}}$ represents the mean value of the bioimpedance signal in position $x$, where $x \in\{1,2,3\}$. The obtained results are depicted in Fig. 8. As it can be seen from Fig. 8c, the lowest overall error occurs between position 3 and position 1 , while the highest overall error occurs between position 1 and position 2, as shown in Fig. 8a.

For calculating parameters such as LVET and PEP we use two different positions (Position 1 and 2). These positions are selected due to the highest obtained error (i.e., worst case scenarios). Characteristic ICG parameters along with the heart rate (HR) of each subject are depicted in Fig. 9. HR is calculated from the ECG signal obtained from our device.

We have done an evaluation of the computational complexity of our algorithms, and we have concluded that we need just between $40 \%$ and $50 \%$ of the duty cycle of the CPU power in the STM32 micro-controller. As we are processing our signals and extracting relevant parameters from them, we use just $0.1 \%$ of the duty cycle of the Radio for sending results such as $Z_{0}, L V E T, P E P, H R$. Therefore, considering the results from the Table I, for our 710mAh battery, we get 106 hours of operation on a single battery charge.

\section{CONCLUSIONS AND FUTURE WORK}

In this paper, we have presented a touch-based ultra-low power portable device which allows real-time beat-to-beat ICG and ECG signals acquisition by touch. For our $710 \mathrm{mAh}$ battery, assuming a continuous monitoring (the worst case with $50 \%$ of duty cycle of STM32L151 and $1 \%$ of duty cycle of Radio), we can reach 106 hours, thus allowing for 4 days of operation on a single battery charge. We have

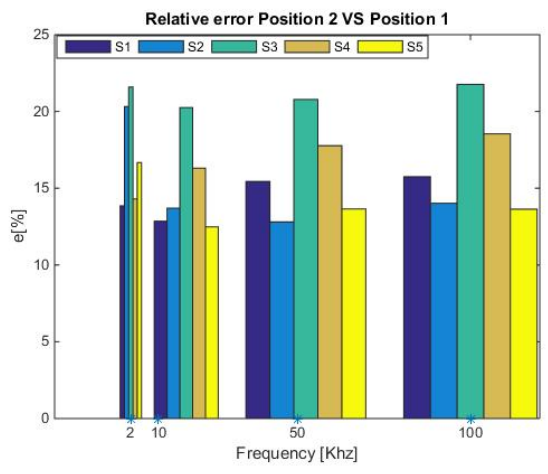

(a) $e_{21}$

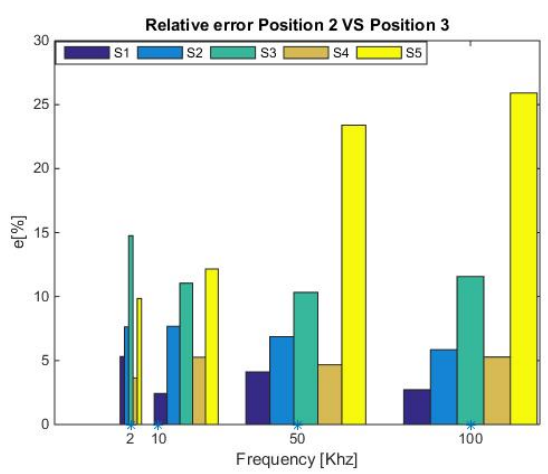

(b) $e_{23}$

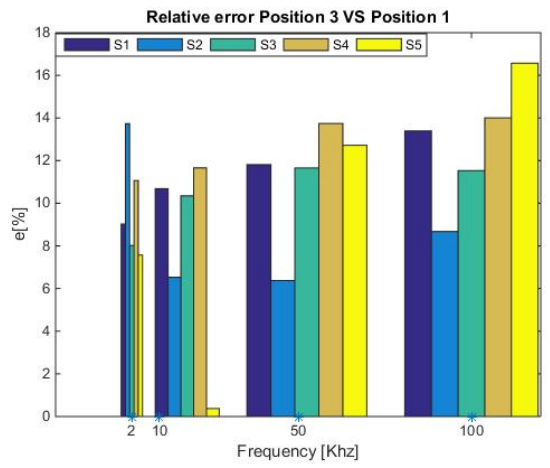

(c) $e_{31}$

Fig. 8: Relative error of bioimpedance value between different positions

demonstrated that there is a strong correlation $(r=85 \%)$ between signals coming from our device and those obtained using the traditional electrode configuration setup. In the worst-case situation, the obtained error is always below $20 \%$. Therefore, slight displacement due to hands shaking does not impact much the obtained results. Acquiring ECG and ICG signal simultaneously from a portable device gives a possibility of estimating hemodynamic parameters, as present in the paper. These parameters can be transmitted wirelessly to a physician for further review in order to prevent possible attacks. Obtaining biomedical signals by means of the hand-held ultra-low power devices offers portability and the option of managing complex patients in outpatient settings. At the same time, these devices greatly simplify the work of 


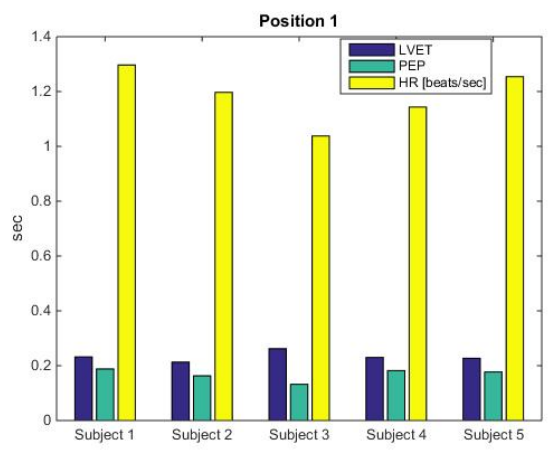

(a) Position 1

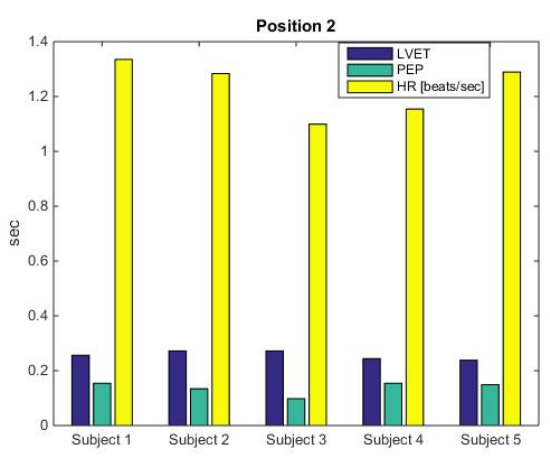

(b) Position 2

Fig. 9: Characteristic ICG parameters along with the HR of each subject for the worst-case positions

hospital physicians in healthcare, as they can be easily used autonomously by patients. As for the future work, we are planning to expand our study on a larger number of subjects and compare our results to those obtained from some already existant ICG system.

\section{ACKNOWLEDGMENT}

This work has been partially supported by the Hasler Foundation (project no. 15048), and RTD project ObeSense (no. 20NA21 143081) evaluated by the Swiss NSF and funded by Nano-Tera.ch with Swiss Confederation financing.

\section{REFERENCES}

[1] A. S. Go et al., "Executive Summary: Heart Disease and Stroke Statistics-2013 Update: A Report From the American Heart Association," Circulation, vol. 127, no. 1, pp. 143-152, Jan. 2013.

[2] S. I. Chaudhry et al., "Patterns of weight change preceding hospitalization for heart failure." Circulation, vol. 116, no. 14, pp. 1549-54, Oct. 2007.

[3] B. A. Borlaug and D. A. Kass, "Invasive hemodynamic assessment in heart failure." Heart failure clinics, vol. 5, no. 2, pp. 217-28, Apr. 2009.

[4] B. F. Geerts et al., "Methods in pharmacology: measurement of cardiac output." British journal of clinical pharmacology, vol. 71, no. 3, pp. 316-30, Mar. 2011.

[5] [Online]. Available: http://lsertercacom24.soup.io/post/432586304/ 3D-Anatomy-Models-for-Animation-Visualization-Be

[6] R. Belardinelli et al., "Comparison of impedance cardiography with thermodilution and direct fick methods for noninvasive measurement of stroke volume and cardiac output during incremental exercise in patients with ischemic cardiomyopathy," The American Journal of Cardiology, vol. 77, no. 15, pp. 1293-1301, Jun. 1996.
[7] W. T. Abraham, "Prospective evaluation of cardiac decompensation in patients with heart failure by Impedance Cardiography Test: the PREDICT MulticenterTrial," Circulation, vol. 110, no. suppl III, 2004.

[8] S. Weyer et al., "Bioelectrical impedance spectroscopy as a fluid management system in heart failure." Physiological measurement, vol. 35, no. 6, pp. 917-30, Jun. 2014.

[9] J. A. McFetridge-Durdle et al., "Ambulatory impedance cardiography in hypertension: a validation study." European journal of cardiovascular nursing : journal of the Working Group on Cardiovascular Nursing of the European Society of Cardiology, vol. 7, no. 3, pp. 204-13, Sep. 2008.

[10] G. Gielerak et al., "The usefulness of impedance cardiography for predicting beneficial effects of cardiac rehabilitation in patients with heart failure." BioMed research international, vol. 2013, p. 595369, Jan. 2013.

[11] A. Sherwood and J. R. Turner, "Hemodynamic responses during psychological stress: implications for studying disease processes." International journal of behavioral medicine, vol. 2, no. 3, pp. 193-218, Jan. 1995.

[12] "Philips." [Online]. Available: http://www.medical.philips.com/

[13] "CAS Medical LIFEGARD II." [Online]. Available: http://www. medwrench.com

[14] S. Weyer et al., "Development of a wearable multi-frequency impedance cardiography device." Journal of medical engineering \& technology, vol. 39, no. 2, pp. 131-7, Feb. 2015.

[15] I. Journals, P. C. Choudhari, and D. M. S. Panse, "Denoising of Radial Bioimpedance Signals using Adaptive Wavelet Packet Transform and Kalman Filter," Mar. 2015.

[16] V. K. Pandey and P. C. Pandey, "Wavelet Based Cancellation of Respiratory Artifacts in Impedance Cardiography," in 2007 15th International Conference on Digital Signal Processing. IEEE, Jul. 2007, pp. 191194.

[17] T. Sebastian et al., "Wavelet based denoising for suppression of respiratory and motion artifacts in impedance cardiography," pp. 501-504, 2011.

[18] "ADS1291 - Medical Analog Front End - Analog Front End (AFE) - Description \& parametrics." [Online]. Available: http: //www.ti.com/product/ads1291

[19] "STM32L1 Series - STMicroelectronics." [Online]. Available: http: $/ /$ www.st.com/stm3211

[20] [Online]. Available: https://www.nordicsemi.com/eng/Products/ Bluetooth-Smart-Bluetooth-low-energy/nRF8001

[21] Y. Sun, K. L. Chan, and S. M. Krishnan, "ECG signal conditioning by morphological filtering," Computers in Biology and Medicine, vol. 32, no. 6, pp. 465-479, Nov. 2002.

[22] M. S. Mialich, J. M. F. Sicchieri, and A. A. J. Junior, "Analysis of Body Composition: A Critical Review of the Use of Bioelectrical Impedance Analysis," International Journal of Clinical Nutrition, vol. 2, no. 1, pp. 1-10, Jan. 2014. [Online]. Available: http: //pubs.sciepub.com/ijcn/2/1/1/index.html

[23] X. Hu et al., "Adaptive Filtering and Characteristics Extraction for Impedance Cardiography," Journal of Fiber Bioengineering and Informatics, vol. 7, no. 1, pp. 81-90.

[24] J. M. Solà i Carós, "Continuous non-invasive blood pressure estimation," 2011.

[25] W. G. Kubicek et al., "Development and evaluation of an impedance cardiac output system." Aerospace medicine, vol. 37, no. 12, pp. 120812, Dec. 1966.

[26] S. H. Thomas, "Impedance cardiography using the Sramek-Bernstein method: accuracy and variability at rest and during exercise." British journal of clinical pharmacology, vol. 34, no. 6, pp. 467-76, Dec. 1992.

[27] U. G. Kyle et al., "Bioelectrical impedance analysis-part I: review of principles and methods." Clinical nutrition (Edinburgh, Scotland), vol. 23, no. 5, pp. 1226-43, Oct. 2004.

[28] M. A. Paulo de Carvalho et. al, Rui Pedro Paiva, Jorge Henriques, "CISUC - Robust Characteristic Points for ICG: Definition and Comparative Analysis."

[29] J. Pan and W. J. Tompkins, "A real-time QRS detection algorithm." IEEE transactions on bio-medical engineering, vol. 32 , no. 3, pp. 230 6, Mar. 1985.

[30] A. Gupta et al., "Bioelectrical Impedance: A Future Health Care Technology," IJCA Proceedings on 4th International IT Summit Confluence 2013 - The Next Generation Information Technology Summit, vol. Confluence, no. 1, pp. 21-25. 